\title{
Aktiver Scheinwerfer mit DMD-Technologie zur Erzeugung vollständiger Lichtverteilungen
}

\author{
M. Knöchelmann, Dr.-Ing. A. Wolf, Dr.-Ing. G. Kloppenburg, \\ Prof. Dr.-Ing. R. Lachmayer, \\ Leibniz Universität Hannover, Institut für Produktentwicklung und \\ Gerätebau
}

\section{Kurzfassung}

In diesem Beitrag wird der Prototyp eines aktiven Scheinwerfers vorgestellt, der durch den Einsatz eines Mikrospiegelarrays (DMD) hochaufgelöste Lichtverteilungen generieren kann. Mit der eingesetzten verzeichnenden Projektionsoptik wird das Aspektverhältnis entsprechende einer KFZ-Lichtverteilung aufgeweitet und ein mittiger Hotspot mit hoher Beleuchtungsstärke erzeugt. Dadurch kann der Nutzungsgrad der Mikrospiegel und damit der Systemwirkungsgrad im Vergleich zu bisherigen Prototypen deutlich gesteigert werden, was anhand einer typischen Fernlichtverteilung gezeigt wird. Basierend auf der optischen Simulation werden die Vor- und Nachteile der verzeichnenden Optik diskutiert und abschließend Ergebnisse der photometrischen Vermessung des Prototyps vorgestellt.

\footnotetext{
Abstract

In this paper we present the prototype of an active headlamp that can generate high-resolution light distributions by using a micromirror array (DMD). A distortive projection optics is used to widen the aspect ratio according to a headlamp light distribution and to generate a central hotspot with high illuminance. As a result, the degree of utilization of the micromirror and thus the system efficiency compared to previous prototypes can be significantly increased, as shown by a typical high beam distribution. Based on the optical simulation, the advantages and disadvantages of the distortive optics are discussed and finally results of the photometric measurement of the prototype are presented.
} 


\section{Einleitung}

Mit neuen Scheinwerfertechnologien wie Matrix-LED Systemen können unter anderem eine Blendung des Gegenverkehrs vermieden und Gefahrenstellen markiert werden. Durch den Technologiesprung zu hochauflösenden Scheinwerfern können diese Lichtfunktionen präziser umgesetzt und der Funktionsumfang erweitert werden, beispielsweise um Projektionen auf die Straße.

Für hochauflösende Scheinwerfer werden aktuell verschiedene Technologien diskutiert [1] - [6], eine klare Entscheidung für oder gegen eine Technologie für die Serienentwicklung ist bisher nicht gefallen. Eine mögliche Umsetzung hochauflösender Scheinwerfer basiert auf der DMD-Technologie (Digital Micromirror Device). Diese aus der Videoprojektion stammende Technik hat im Vergleich zu anderen Technologien eine sehr hohe Auflösung und ist sehr schnell schaltbar. Nachteilig ist, dass DMDs bisher nur mit einem von der KFZ-Lichtverteilung stark abweichenden Seitenverhältnis verfügbar sind. Eine klassische, verzeichnungsfreie Projektionsoptik bildet den DMD so ab, dass eine homogene Lichtverteilung entsteht. Daher muss im gesamten Winkelbereich der Lichtverteilung die maximale Hotspot-Beleuchtungsstärke vorgehalten werden, was im Randbereich zu einer dauerhaft hohen Absorptionsrate des Lichtes und einer niedrigen Systemeffizienz führt. Sinnvoller ist es einen Teil des Lichtstroms von den Rändern zu der Mitte der Lichtverteilung umzuverteilen und so die Effizienz deutlich zu steigern.

Eine vielversprechende Lösung ist die Verwendung einer verzeichnenden Projektionsoptik mit asphärischen Linsen, die im Vergleich zu einer konventionellen abbildenden Optik eine Verbreiterung des Öffnungswinkels des Scheinwerfers ermöglicht und gleichzeitig einen zentralen Hotspot erzeugt. Damit ist es möglich, eine vollständige Scheinwerfer-Lichtverteilung mit einem einzigen optischen System zu erzeugen. Gleichzeitig kann der Wirkungsgrad gegenüber nicht-verzeichnenden Systemen signifikant gesteigert werden. In diesem Beitrag werden deshalb die Herausforderungen betrachtet, die sich bei der Entwicklung eines solchen hochauflösenden Scheinwerfers mit DMD-Technologie ergeben. Die Validierung erfolgt anhand von Scheinwerfer-Prototypen, die auf der rotationssymmetrischen Verzeichnung basieren.

\section{Systembeschreibung}

Mit vollständig adaptiven und hochauflösenden Scheinwerfersystemen, wie dem hier beschriebenen DMD-Scheinwerfer, können neuartige Funktionalitäten umgesetzt werden. Mit der Projektion von Symbolen direkt auf die Fahrbahnoberfläche entsteht eine völlig neue Möglichkeit der Kommunikation im Verkehrsraum, die auch insbesondere im Kontext autonomer 
Fahrzeuge an Bedeutung gewinnen wird. Auf dem Weg zu teilautonomen und vollständig autonomen Fahrfunktionen spielen die Erkennung und Interpretation der Umgebung und der aktuellen Verkehrssituation eine entscheidende Rolle. Zukünftige Scheinwerfer können genutzt werden, um Sensorik zu integrieren, oder durch die Projektion von Mustern die Umgebung für Kamerasysteme besser sichtbar zu machen.

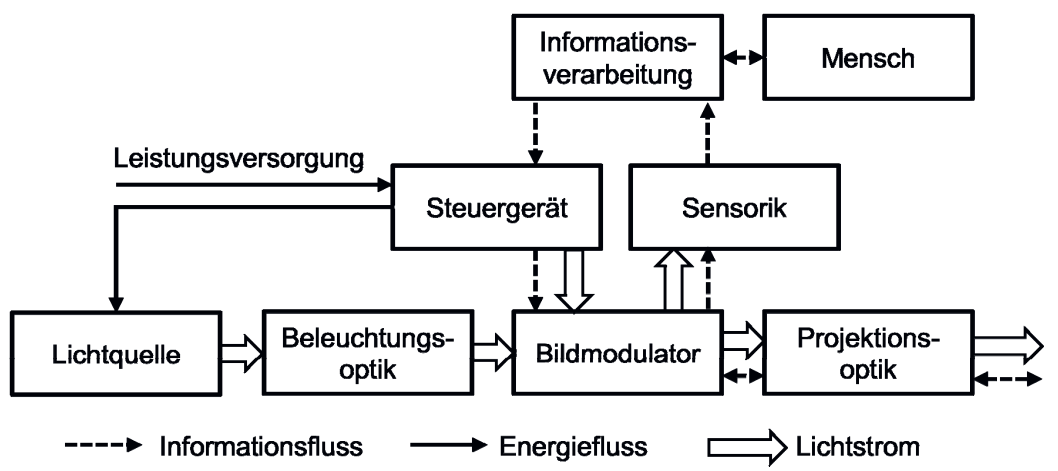

Bild 1: Elemente eines hochauflösenden Scheinwerfers nach [7]

Aktuelle Scheinwerfer werden als mechatronische Systeme beschrieben, die Signale einer zentralen Fahrzeugsteuerung mit Aktoren umsetzen und eine begrenzte Zahl zuvor definierter Lichtfunktionen anzeigen. Um die neuen Funktionalitäten zukünftiger Scheinwerfer zu beschreiben, muss diese Betrachtung erweitert werden. In Bild 1 sind die Elemente eines hochauflösenden Scheinwerfers sowie der Energie- und Informationsfluss dargestellt. Da der Lichtstrom durch das System wichtig ist, wird dieser in der Darstellung abweichend von der VDI 2206 [7] statt des Stoffflusses dargestellt. Die Besonderheit des dargestellten Systems ist der Informationsaustausch des Scheinwerfers mit der Umgebung. Die durch den Bildmodulator erzeugte Information wird mittels der Projektionsoptik auf die Straße projiziert. Die Optik kann gleichzeitig dazu genutzt werden aus einem großen Winkelbereich Informationen wie die Straßentopologie oder die Beleuchtungssituation zu erfassen.

In diesem Beitrag wird die erstgenannte Möglichkeit der Informationsdarstellung mittels hochauflösender Fahrzeugscheinwerfer betrachtet. 


\section{Analyse}

Das Ziel des beschriebenen Vorhabens ist es vollständige KFZ-Lichtverteilungen mit einem DMD-basierten Scheinwerfer zu erzeugen. In diesem Kapitel wird aufgezeigt, welche Herausforderungen dabei auftreten.

Für diese Analyse wird zuerst eine Fernlichtverteilung als Grundlage definiert. Diese Lichtverteilung stellt den Ausgangspunkt für alle weiteren Lichtfunktionen dar, welche durch lokales reduzieren der Lichtmenge erzeugt werden können. Die Effizienz des Systems wird basierend auf der Fernlichtteilung aus Bild 2 durchgeführt. Andere Betriebszustände wie Abblendlicht oder das Ausblenden von Verkehrszeichen [8] und anderen Verkehrsteilnehmern führen durch die subtraktive Bilderzeugung des DMDs zu einem geringeren Systemwirkungsgrad.

\subsection{Lichtverteilung}

In Bild 2 ist die Beleuchtungsstärkeverteilung eines beispielhaften Scheinwerfers gezeigt, wobei das HID-Fernlicht sowie ein halogenbasiertes Zusatzfernlicht aktiviert sind. Im Zentrum wird die maximale Beleuchtungsstärke von 156 Ix erreicht, der Gesamtlichtstrom der Verteilung beträgt $1440 \mathrm{Im}$.

Für die weitere Betrachtung wird die Lichtverteilung auf den relevanten Winkelbereich $\pm 25^{\circ}$ horizontal und $+5 /-10^{\circ}$ vertikal reduziert, wo etwa $86 \%$ des Gesamtlichtstroms auftreten.

KFZ-Lichtverteilungen umfassen einen schmalen vertikalen und breiten horizontalen Winkelbereich und weisen einen zentralen Hotspot mit hoher Beleuchtungsstärke auf. Das Aspektverhältnis, also der Quotient aus maximaler horizontaler und vertikaler Ausdehnung der Lichtverteilung, liegt bei etwa 4:1. Im Hotspot ist eine Beleuchtungsstärke gewünscht, die am Rand der Lichtverteilung um den Faktor 100 und mehr geringer ist. Das Seitenverhältnis und die inhomogene Beleuchtungsstärkeverteilung sind Eigenschaften einer KFZ-Lichtverteilung, die sich von der Hauptanwendung von DMDs in Videoprojektoren unterscheiden, wo das Aspektverhältnis beispielsweise $4: 3$ oder $16: 10$ beträgt und eine möglichst homogene Beleuchtungsstärkeverteilung gefordert ist. 


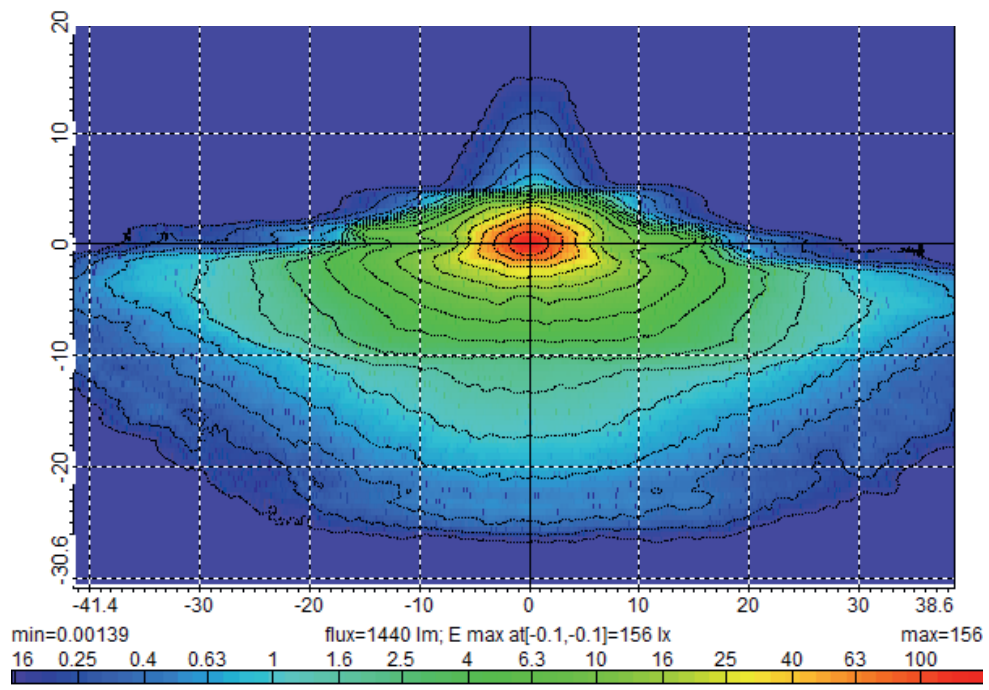

Bild 2: Beispielhafte Lichtverteilung eines Fernlichts

\section{Herausforderung 1:}

Anpassen des Aspektverhältnisses des DMDs auf das Verhältnis von KFZ-Lichtverteilungen

\section{Herausforderung 2:}

Erzeugen einer inhomogenen Beleuchtungsstärkeverteilung mit zentralem Hotspot

Der maximal erreichbare Kontrast einer Projektion ist eine wichtige Eigenschaft für die Bewertung von Videoprojektoren und liegt für DMD-Projektoren bei etwa 2000:1. Eine Anforderung an den Kontrast für Lichtverteilungen kann aus der ECE 112 [9] abgeleitet werden, in der für eine Abblendlichtverteilung über der Hell-Dunkel Grenze eine maximale Beleuchtungsstärke von 0,4 Ix gefordert wird. Für die definierte Fernlichtverteilung muss der Scheinwerfer einen Kontrast von 309:1 erreichen, also deutlich weniger als bei Videoprojektoren. 


\subsection{Systemwirkungsgrad}

Die Bilderzeugung mit einem DMD erfolgt subtraktiv, das bedeutet, dass bei einer homogenen Beleuchtung des DMDs viele Pixel dauerhaft stark gedimmt werden müssen. Dies hat eine starke Reduktion des Systemwirkungsgrades zur Folge. Der Systemwirkungsgrad setzt sich bei einem DMD-Scheinwerfer mit subtraktiver Bilderzeugung aus dem optischen Wirkungsgrad und dem Nutzungsgrad des DMDs zusammen. Der Nutzungsgrad gibt an wie hoch das Verhältnis der An und Aus-Positionen der Spiegel des DMDs ist. Nachfolgend wird der Nutzungsgrad für die oben gezeigte Lichtfunktion nach der Notation von Gut [10] hergeleitet.

Die Beschreibung der Lichtfunktion erfolgt anhand der Lichtstärkematrix $\mathrm{A}_{\mathrm{I}}$ der Lichtfunktion.

$$
\mathrm{A}_{\mathrm{I}}=\left[\begin{array}{ccc}
\mathrm{I}_{\mathrm{F}, 11} & \cdots & \mathrm{I}_{\mathrm{F}, 1 \mathrm{n}} \\
\vdots & \ddots & \vdots \\
\mathrm{I}_{\mathrm{F}, \mathrm{m} 1} & \cdots & \mathrm{I}_{\mathrm{F}, \mathrm{mn}}
\end{array}\right]
$$

Der Lichtstrom einer Lichtfunktion $\phi_{\mathrm{V}, \mathrm{F}}$ ergibt sich über das Integral der Winkelbereiche $u^{\prime}$ und $v^{\prime}$ (Definition der Winkel in Bild 4):

$$
\phi_{\mathrm{V}, \mathrm{F}, i j}=\int_{-v^{\prime}}^{v^{\prime}} \int_{-u^{\prime}}^{u^{\prime}} \mathrm{I}_{i j} \mathrm{~d} u^{\prime} \mathrm{d} v^{\prime} .
$$

Die gesamte Lichtstrommatrix hat die folgende Form:

$$
\mathrm{A}_{\phi}=\left[\begin{array}{ccc}
\phi_{\mathrm{V}, \mathrm{F}, 11} & \cdots & \phi_{\mathrm{V}, \mathrm{F}, 1 n} \\
\vdots & \ddots & \vdots \\
\phi_{\mathrm{V}, \mathrm{F}, m 1} & \cdots & \phi_{\mathrm{V}, \mathrm{F}, m n}
\end{array}\right] .
$$

Das Funktionsprinzip des DMDs beruht auf einer subtraktiven Bilderzeugung. Licht, das auf den DMD trifft wird entweder in die Projektionsoptik gelenkt, oder in einer Lichtfalle absorbiert. Bei einer homogenen Beleuchtung des DMD bedeutet dies, dass der notwendige Lichtstrom der Lichtquelle $\phi_{\mathrm{V}, \mathrm{Q}}$ von dem maximalen Teillichtstrom $\phi_{\mathrm{V}, \mathrm{F}, m n}$ abhängt. Dieser Teillichtstrom muss bei einer homogenen Beleuchtung des DMDs auf dem gesamten DMD vorgehalten werden.

$$
\phi_{\mathrm{V}, \mathrm{Q}}=\frac{n \cdot m}{\eta_{\mathrm{BO}} \cdot \eta_{\mathrm{DMD}} \cdot \eta_{\mathrm{PO}}} \max \left[\begin{array}{ccc}
\phi_{\mathrm{V}, \mathrm{F}, 11} & \cdots & \phi_{\mathrm{V}, \mathrm{F}, 1 n} \\
\vdots & \ddots & \vdots \\
\phi_{\mathrm{V}, \mathrm{F}, m 1} & \cdots & \phi_{\mathrm{V}, \mathrm{F}, m n}
\end{array}\right]
$$

Der Wirkungsgrad der Beleuchtungsoptik $\eta_{\text {во }}$ setzt sich aus dem optischen Wirkungsgrad des Linsensystems und dem Anteil des Lichts, das von der Lichtquelle mit der Optik aufgefangen werden kann, zusammen. Der Wirkungsgrad des DMDs $\eta_{D M D}$ enthält die Reflexions- und Transmissionseigenschaften des Spiegelmaterials und der Scheibe vor dem Spiegelarray. Es ist außerdem ein Faktor zur Berücksichtigung des Beleuchtungswinkels enthalten. Der Wirkungsgrad der Projektionsoptik $\eta_{\mathrm{PO}}$ hängt von der Anzahl, Qualität und Beschichtung der Linsen $a b$. 
Aus $\phi_{\mathrm{V}, \mathrm{Q}}$ ergibt sich mit

$$
\phi_{\mathrm{V}, \mathrm{F}}=\sum_{i=1}^{m} \sum_{j=1}^{n} \phi_{\mathrm{V}, \mathrm{F}, i j}
$$

der optische Systemwirkungsgrad $\eta_{\text {Sys }}$.

$$
\eta_{\mathrm{Sys}}=\frac{\phi_{\mathrm{V}, \mathrm{F}}}{\phi_{\mathrm{V}, \mathrm{Q}}}
$$

Gut [10] definiert einen Nutzungsgrad K, um die Auswirkung der subtraktiven Bilderzeugung des DMDs mit anderen Technologien vergleichen zu können. Der Nutzungsgrad $K_{F}$ wird für eine spezielle Lichtfunktion bestimmt, indem für jeden Spiegel des DMD das Verhältnis zwischen An- und Aus-Position bestimmt wird und anschließend über den gesamten DMD gemittelt wird.

$$
\mathrm{K}_{\mathrm{F}}=\frac{\sum_{i=1}^{m} \sum_{j=1}^{n} \phi_{\mathrm{V}, \mathrm{F}, i j}}{\max \left(\mathrm{A}_{\phi}\right) \cdot m \cdot n}
$$

Zusammen mit den optischen Wirkungsgraden der Einzelkomponenten kann aus dem Nutzungsgrad ebenfalls der optische Systemwirkungsgrad $\eta_{\text {Sys }}$ bestimmt werden.

$$
\eta_{\text {Sys }}=\frac{\phi_{\mathrm{V}, \mathrm{F}}}{\phi_{\mathrm{V}, \mathrm{Q}}}=\mathrm{K}_{\mathrm{F}} \cdot \eta_{\mathrm{BO}} \cdot \eta_{\mathrm{DMD}} \cdot \eta_{\mathrm{PO}}
$$

Für die zuvor definierte Fernlichtverteilung ergibt sich für den Winkelbereich $\pm 25^{\circ}$ horizontal und $+5 /-10^{\circ}$ vertikal ein Nutzungsgrad von $K_{F}=5,2 \%$. Gut [10] berechnet für eine andere Fernlichtverteilung in einem Winkelbereich von $\pm 20^{\circ}$ horizontal und $\pm 10^{\circ}$ vertikal einen Nutzungsgrad von $\mathrm{K}_{\mathrm{F}}=8,5 \%$. Für andere Lichtfunktionen reduziert sich der Wert noch weiter, da in der Fernlichtverteilung der höchste Lichtstrom genutzt wird. Um den Energiebedarf und die Wärmeentwicklung der Lichtquelle zu begrenzen, muss für einen Einsatz eines DMDs für vollständige Lichtverteilungen der Nutzungsgrad deutlich erhöht werden.

Herausforderung 3: Erhöhung des Nutzungsgrades

DMDs, die für den Einsatz im Fahrzeug geeignet sind, haben im Vergleich zu anderen Lichtmodulatoren eine sehr kleine Fläche. Die Anforderungen an die Leuchtdichte der Lichtquelle sind daher besonders hoch. Auf die Auswahl und Auslegung der Lichtquelle wird in diesem Beitrag aber nicht der Fokus gelegt. 


\section{Synthese des optischen Systems}

Für die analysierten Herausforderungen existieren bereits Lösungen, die teilweise als Prototypen umgesetzt sind. Tabelle 1 gibt einen Überblick über mögliche Lösungen und ordnet den Stand der Forschung zu. Anschließend wird in diesem Kapitel die Auslegung der Projektionsoptik beschrieben und Simulationsergebnisse dazu vorgestellt.

Tabelle 1: Optische Konzepte für vollständige Lichtverteilungen

\begin{tabular}{|l|c|c|c|}
\hline & $\begin{array}{c}\text { Aspektverhältnis } \\
\text { anpassen }\end{array}$ & $\begin{array}{c}\text { Hotspot } \\
\text { erzeugen }\end{array}$ & $\begin{array}{c}\text { Nutzungsgrad } \\
\text { erhöhen }\end{array}$ \\
\hline $\begin{array}{l}\text { Variation der Abbildungsbedingungen } \\
\text { der Projektionsoptik } \\
\text { [11] - [15] }\end{array}$ & $\mathrm{X}$ & $\mathrm{X}$ \\
\hline $\begin{array}{l}\text { Inhomogene Beleuchtung des DMD } \\
\text { [11], [12], [16] }\end{array}$ & & $\mathrm{X}$ & $\mathrm{X}$ \\
\hline Umfeldverspiegelung [13] & $\mathrm{X}$ & & \\
\hline $\begin{array}{l}\text { Nutzung des Absorberlichtstroms } \\
\text { [17] - [19] }\end{array}$ & & & $\mathrm{X}$ \\
\hline Einsatz einer Pixellichtquelle [20], [21] & & & $\mathrm{X}$ \\
\hline
\end{tabular}

Die Variation der Abbildungsbedingungen der Projektionsoptik ist ein vielversprechendes Konzept, das bereits von mehreren Forschergruppen untersucht und teilweise als Prototyp umgesetzt wurde. Günther [11] und Bhakta [12] nutzen für die Aufweitung des Aspektverhältnisses eine Projektionsoptik mit Zylinderlinsen.

Zur Erzeugung eines mittigen Hotspots kann der DMD inhomogen beleuchtet werden. Durch diese Vormodellierung auf dem DMD kann die Systemeffizienz im Vergleich zu einer homogenen Ausleuchtung des DMD deutlich erhöht werden. Bestenfalls ist kein weiteres Dimmen einzelner Pixel mehr durch den DMD notwendig.

Als weiteren Ansatz, um das Aspektverhältnis der Lichtverteilung anzupassen, schlägt Kauschke [13] vor, das Umfeld des DMD zu verspiegeln. Aufgrund der möglichen Abschattung durch den schrägen Lichteinfall und die Schwierigkeit einen Spiegel in der gleichen Ebene zu platzieren wie die DMD-Mikrospiegel, verfolgt Kauschke dieses Konzept selbst nicht weiter. Statt das Licht ausgeschalteter oder gedimmter Pixel auf einen Absorber zu lenken, kann dieses für andere Zwecke, zum Beispiel zur Generation einer Grundlichtverteilung, genutzt werden [17] - [19]. Kauschke [13] gibt zu beachten, dass eine wechselseitige Verknüpfung 
zwischen dem Nutzlicht und dem Absorberlicht besteht. Er sieht die Nachteile überwiegen und verfolgt die Nutzung des Absorberlichts nicht weiter.

Wird eine Lichtquelle eingesetzt, die aus mehreren schaltbaren Einzellichtquellen besteht, z.B. ein LED Array, kann die Beleuchtung des DMDs an die aktuelle Lichtverteilung angepasst werden [20], [21]. Mit diesem Ansatz ist eine deutliche Steigerung des Nutzungsgrades zu erwarten.

Da die Variation der Abbildungsbedingungen der Projektionsoptik auf alle der Herausforderungen einen positiven Einfluss haben kann, wird dieses Konzept weiter verfolgt. Für die Auslegung der Projektionsoptik wird das Teilsystem (DMD $\rightarrow$ Projektionsoptik $\rightarrow 25 \mathrm{~m}$ Wand) genauer betrachtet.

Der Abbildungsmaßstab eines optischen Systems ist definiert als Verhältnis zwischen der Bildgröße und der realen Gegenstandsgröße. Um den Einfluss des Abbildungsmaßstabes zu verdeutlichen, wird aus der Lichtstärkematrix $\mathrm{I}_{\mathrm{F}}$ die Beleuchtungsstärkematrix $\mathrm{E}_{25 \mathrm{~m}}$ abgeleitet. KFZ-Lichtverteilungen werden üblicherweise auf einer $r=25 \mathrm{~m}$ entfernten Messwand bewertet. Bei senkrechtem Lichteinfall gilt:

$$
\mathrm{E}=\frac{\mathrm{I}_{\mathrm{F}}}{\mathrm{r}^{2}} .
$$

Der Zusammenhang zwischen der Beleuchtungsstärkematrix der Messwand $\mathrm{E}_{25 \mathrm{~m}}$ und der Beleuchtungsstärkematrix des DMD $\mathrm{E}_{\mathrm{DMD}}$ ist über den Abbildungsmaßstab $\beta$ der Projektionsoptik gegeben:

$$
\beta^{2}=\frac{E_{25 m}}{E_{D M D}} .
$$

In abbildenden optischen Systemen ist der Abbildungsmaßstab konstant über die gesamte Gegenstandsfläche. Das Seitenverhältnis der Abbildung ist identisch zu der des Gegenstandes und die Lichtstärke bleibt ebenfalls konstant. Wird bei der Projektionsoptik gezielt der Abbildungsmaßstab über die Fläche verändert, ergeben sich Vorteile, die für die Darstellung von KFZ-Lichtverteilungen genutzt werden können. 
a)

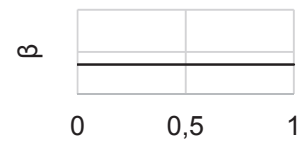

b)

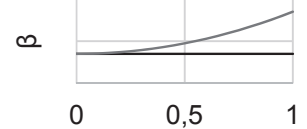

c)

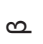

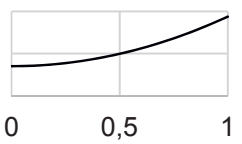

d)

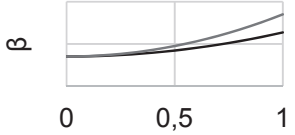

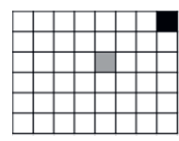
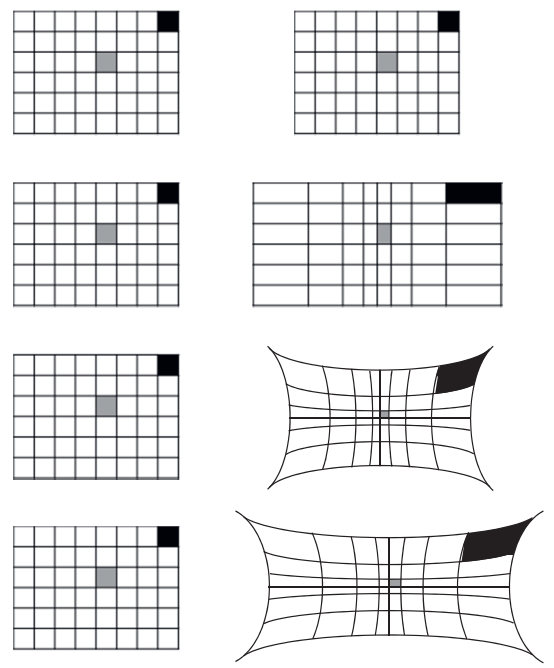

Bild 3: Konzepte für die Projektionsoptik. Abszisse: Relative Position auf dem DMD. Ordinate: Abbildungsmaßstab. Variation des Abbildungsmaßstabes: a) konstant. b) veränderlich in Richtung $x$ oder $y . c$ ) veränderlich in radialer Richtung. d) unterschiedlich veränderlich in Richtungen $x$ und $y$.

In Bild 3 ist dargestellt, welchen Einfluss eine Änderung des Abbildungsmaßstabes hat. Das Ziel des veränderlichen Abbildungsmaßstabes ist es eine homogen beleuchtete Fläche so abzubilden, dass eine inhomogene Abbildung dieser Fläche entsteht. Bild 3 a) zeigt den konstanten Abbildungsmaßstab eines idealen optischen Systems ohne Verzeichnung. Bei b) wird die Abbildung in einer Richtung z.B. durch den Einsatz von Zylinderlinsen gestreckt. Das Aspektverhältnis kann mit diesem Ansatz gezielt beeinflusst werden. An der Größe der abgebildeten Pixel ist ersichtlich, dass sich außerdem ein horizontaler Gradient der Beleuchtungsstärke mit einem Maximum im Bereich der mittigen vertikalen Achse ergibt.

Wie in Bild 3 ersichtlich ist, kann damit das Aspektverhältnis beeinflusst werden und außerdem Lichtstrom von den Rändern der Lichtverteilung zur Mitte umverteilt werden. Der veränderliche Abbildungsmaßstab in radialer Richtung in c) führt zu ähnlichen Effekten. Vorteilhaft gegenüber b) ist, dass ein runder Bereich hoher Beleuchtungsstärke im Zentrum entsteht, der direkt für den Hotspot genutzt werden kann. Das Aspektverhältnis kann in diesem Ansatz nicht unabhängig von der Kurve des Abbildungsmaßstabes verändert werden. Daher ergeben sich 
aus der gewünschten Änderung des Aspektverhältnisses feste Stützpunkte, die bei der Auslegung der Optik beachtet werden müssen. In d) ist der Ansatz mit der höchsten Flexibilität gezeigt, bei dem die Änderung des Abbildungsmaßstabs in $x$ - und y-Richtung unabhängig voneinander bestimmt werden kann.

Um das Potenzial der Variation des Abbildungsmaßstabes zu analysieren, soll eines der Konzepte bei der Auslegung einer Projektionsoptik umgesetzt werden, um anschließend einen Prototypen eines DMD-Scheinwerfers aufbauen.

Der Ansatz c) erlaubt den Einsatz von rotationssymmetrischen Linsen, was Vorteile bei der Simulation, der Fertigung und der späteren algorithmischen Bildkorrektur bietet. Nachfolgend wird daher beschrieben, wie ein optisches System nach Ansatz c) ausgelegt und simuliert werden kann.

\subsection{Auslegung des optischen Systems}

Das Ziel der im Folgenden beschriebenen Auslegung ist es die Kurve des Abbildungsmaßstabes analytisch zu bestimmen und anschließend in einer optischen Simulation umzusetzen. Im Folgenden wird dafür nicht mehr der Abbildungsmaßstab beschrieben, sondern der Zusammenhang zwischen der Position ohne Verzeichnung auf dem DMD und der erwünschten Position mit Verzeichnung auf der Wand.

Im ersten Schritt werden die radialen Maxima der Beleuchtungsstärke bestimmt, damit die geforderte Lichtverteilung dargestellt werden kann. Dafür wird die Lichtverteilung in Polarkoordinaten umgewandelt und für jedes $\mathrm{r}$ die maximale Beleuchtungsstärke $\mathrm{E}(\varphi)$ in Abhängigkeit vom Winkel $\varphi$ bestimmt.

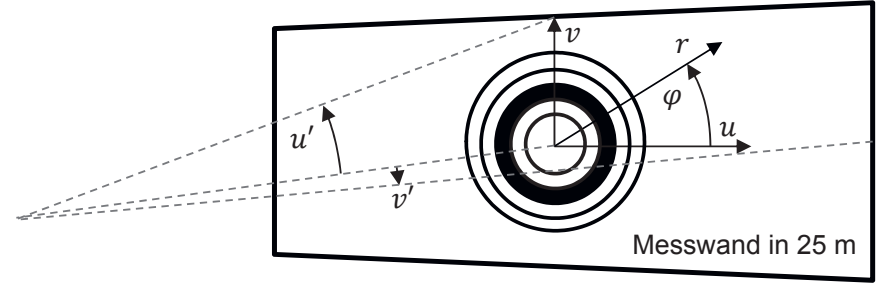

Bild 4: Bestimmung der radialen Maxima der Lichtverteilung

Für jedes Ringsegment kann anschließend der notwendige Lichtstrom $\mathrm{d} \phi\left(\mathrm{r}_{\text {Wand }}\right)$ ermittelt werden:

$$
\mathrm{d} \phi\left(\mathrm{r}_{\text {Wand }}\right)=\mathrm{E}\left(\mathrm{r}_{\text {Wand }}\right) \cdot \pi \cdot \mathrm{dr}_{\text {Wand }}{ }^{2}
$$


Aus dem Lichtstrom jedes Segments wird jetzt die Fläche auf der Wand bestimmt, die das Ringsegment bei einer nicht verzeichnenden Abbildung hätte. Dafür wird eine beliebige konstante Beleuchtungsstärke, hier $\mathrm{E}_{\text {const }}=1 \mathrm{~lx}$, auf der Wand angenommen.

$$
\mathrm{dA}=\mathrm{d} \phi\left(\mathrm{r}_{\text {Wand }}\right) / \mathrm{E}_{\text {const }}
$$

Aus dieser Formel wird anschließend der Radius bestimmt, wobei dieser Radius der Betrachtung ohne Verzeichnung dem Radius $\mathrm{dr}_{\mathrm{DMD}}$ auf dem DMD entspricht. Nach einer Normierung auf den jeweils maximalen Radius ist damit der Zusammenhang zwischen dem Radius $\mathrm{r}_{\text {Wand }}$ und $\mathrm{r}_{\mathrm{DMD}}$ bzw. den Positionen gefunden.

In einer anschließenden optischen Simulation wird ein Linsensystem dahingehend optimiert, dass die geforderte Verzeichnung erreicht wird.

\subsection{Ergebnis der Simulation}

In Bild 5 ist die Kurve der Verzeichnung der simulierten Projektionsoptik dargestellt. Gegenüber einem nicht verzeichnenden optischen System ist die starke Umverteilung des Lichts hin zur Mitte erkennbar.

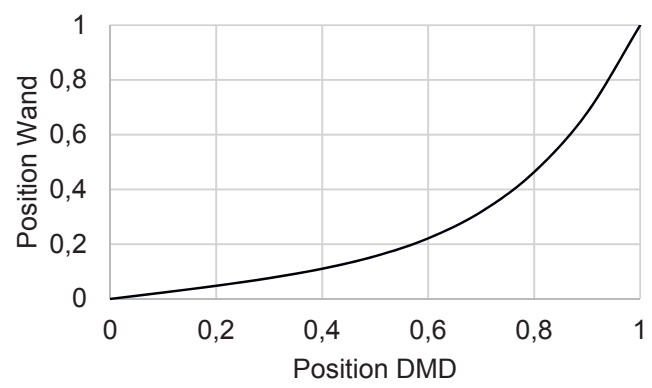

Bild 5: Kurve der Verzeichnung der simulierten Projektionsoptik

In Bild 6 ist ein horizontaler Schnitt durch die Lichtverteilung der simulierten Optik dargestellt. Die starke Verzeichnung der Optik resultiert in hohen Gradienten der Beleuchtungsstärke. Auf der rechten Seite wurden an den Positionen $0^{\circ}, 3^{\circ}$ und $8,3^{\circ}$ jeweils acht Pixel ausgeschaltet, um den erreichbaren Kontrast zu bestimmen. 

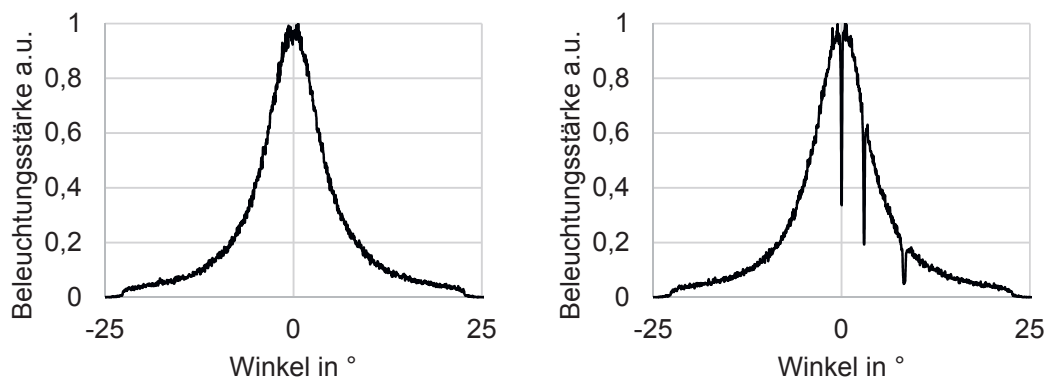

Bild 6: Horizontaler Schnitt durch die simulierte Lichtverteilung der Projektionsoptik. Rechts: Ausblendung von acht Pixeln an drei Positionen

Als Kontrast in Bild 7 ist das Verhältnis der Beleuchtungsstärke mit und ohne deaktivierte Pixel angegeben. Es ist ersichtlich, dass der Kontrast in der Mitte der Verteilung $\left(0^{\circ}\right)$ geringer ist als am Rand. Dies kann durch die Verzeichnung der Optik verursacht werden, da diese Pixel am Rand größer abgebildet als in der Mitte der Verteilung. Um den Einfluss der chromatischen Aberration zu analysieren, wurde die Simulation mit acht deaktivierten Pixeln zusätzlich monochromatisch durchgeführt. Der Kontrast ist in der monochromatischen Simulation etwa viermal so hoch wie in der polychromatischen. Aufgrund des Fertigungsprozesses der Linsen wurde für alle Linsen das Material PMMA gewählt und die Simulation daher nicht hinsichtlich einer geringen chromatischen Aberration optimiert.

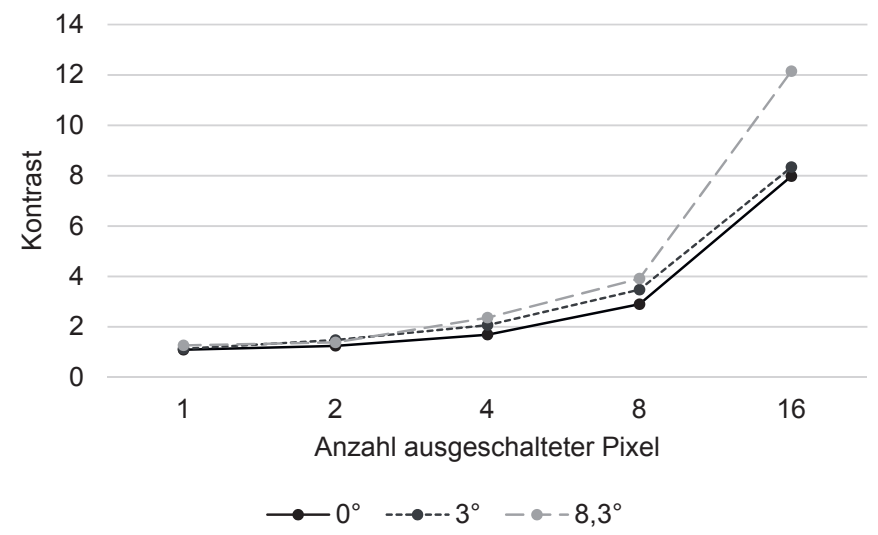

Bild 7: Kontrast in der Simulation an drei Positionen der Lichtverteilung 


\section{Validierung}

Die simulierte Projektionsoptik wurde am IPeG gefertigt ein Prototyp eines hochauflösenden Scheinwerfers aufgebaut (Bild 8). In diesem Kapitel werden die Ergebnisse der photometrischen Vermessung gezeigt und beschrieben. Die Vermessung erfolgte auf einem Fernfeldgoniophotometer in $25 \mathrm{~m}$ Messdistanz.

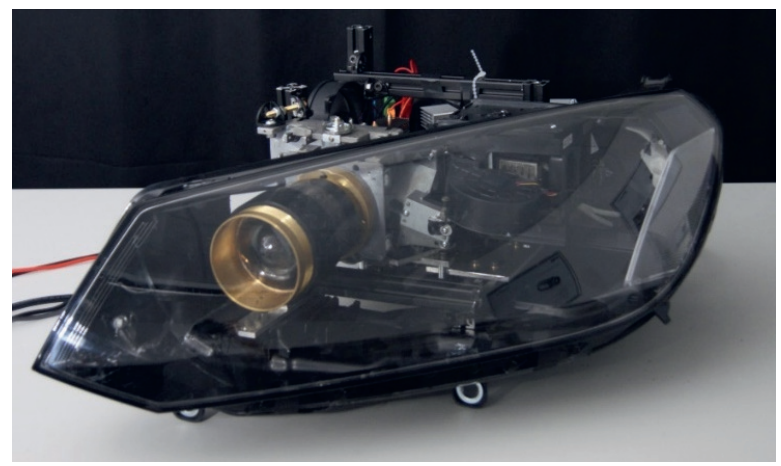

Bild 8: Prototyp im Scheinwerfergehäuse

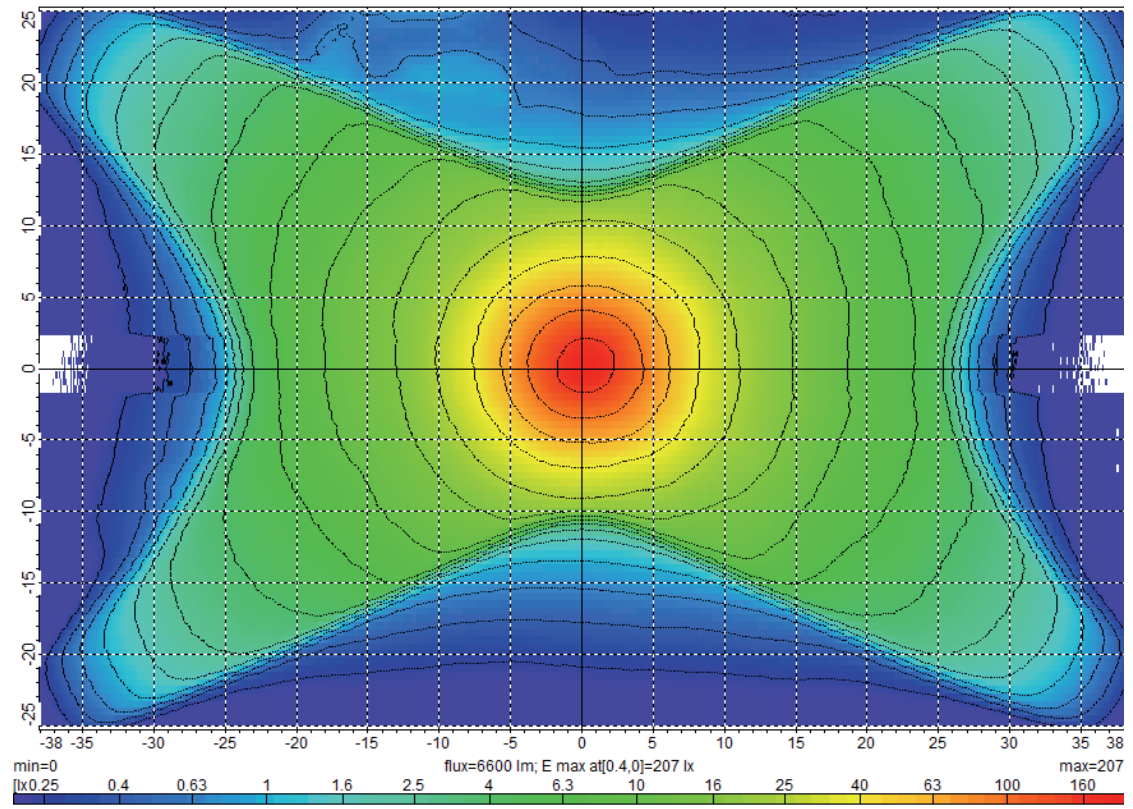

Bild 9: Vollständige Messung der Lichtverteilung des Prototyps 


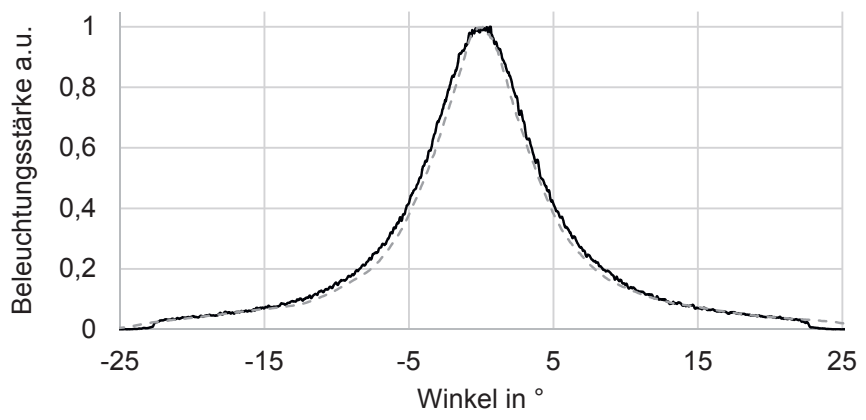

_ Simulation - - - Messung

Bild 10: Vergleich des horizontalen Schnitts der Simulation und Messung im horizontalen Schnitt, normiert auf die maximale Beleuchtungsstärke

Die in Bild 9 gezeigte Lichtverteilung ergibt sich, wenn sich alle Spiegel des DMDs in der AnStellung befinden, also ein weißes Bild projiziert wird. Die maximale Beleuchtungsstärke beträgt $207 \mathrm{Ix}$ im Zentrum der Verteilung und der gesamte Lichtstrom beträgt $6600 \mathrm{Im}$. In einem Rechteck von $\pm 25^{\circ}$ horizontal und $\pm 10^{\circ}$ vertikal treffen $5290 \mathrm{Im}$ auf, was etwa $80 \%$ des Gesamtlichtstroms beträgt. Dieser Bereich kann für die Darstellung von Lichtfunktionen sinnvoll genutzt werden, sodass der Wirkungsgrad mit der vorgestellten Optik maximal $80 \%$ betragen kann. Im Vergleich mit der Simulation zeigt sich die hohe Qualität der Fertigung und Justage der optischen Komponenten (Bild 10).
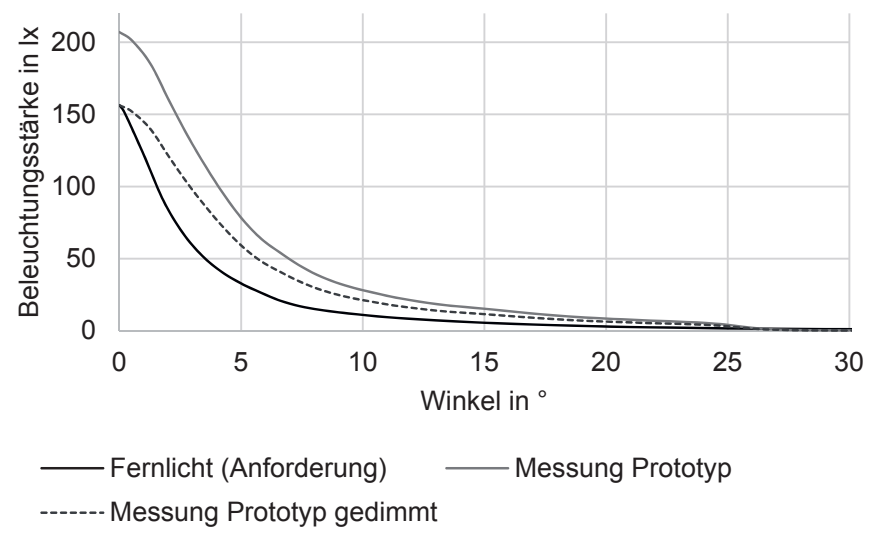

Bild 11: Vergleich der Beleuchtungsstärke des Prototyps mit dem definierten Fernlicht 
In Bild 11 ist die Beleuchtungsstärke des Prototyps der definierten Fernlichtverteilung gegenübergestellt. Der Nutzungsgrad für diese Lichtverteilung beträgt bei voller Leistung des Prototyps 18,9 \%. In diesem Fall kann ohne Einbußen in der Beleuchtungsstärke des Hotspots ein um $\pm 2^{\circ}$ mitschwenkendes Kurvenlicht realisiert werden. Wird der Lampenlichtstrom soweit reduziert, dass der Prototyp genau die geforderte Beleuchtungsstärke im Hotspot erreicht, beträgt der Nutzungsgrad 25,8 \%.

Der theoretische Kontrast des Systems wird mittels Raytracing bestimmt, in dem an drei Positionen der Lichtverteilung Pixel ausgeblendet werden. Die entsprechende Messung am realen System Messung zeigt, dass die Werte der Simulation nicht erreicht werden können. Eine Ursache dafür ist die Unschärfe der Projektionsoptik, die unter anderem durch Fertigungstoleranzen und Justage bedingt ist. Ungewollte Streuung von Licht an der Innenseite des Gehäuses der Optik hat ebenfalls einen negativen Einfluss auf den gemessenen Kontrast. Zusammen mit einer Antireflexbeschichtung der zurzeit nicht beschichteten Linsen lässt sich dieses Potential für eine Optimierung des Systems nutzen.

In der Szene in Bild 12 sind exemplarische einige mögliche Funktionen dargestellt, die sich mit dem DMD-Scheinwerfer umsetzen lassen.

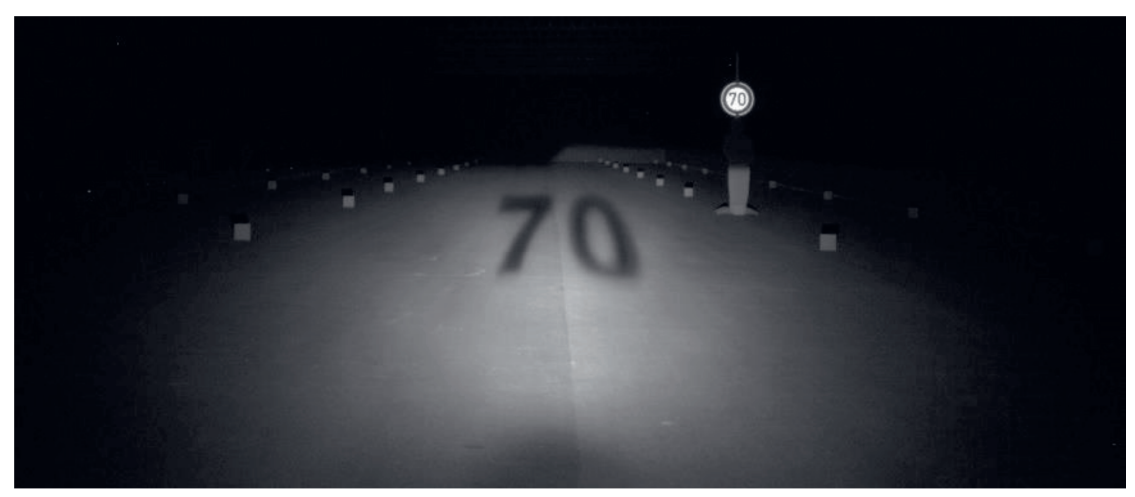

Bild 12: Einsatz des Prototyps zur Informationsprojektion bei Abblendlicht im Lichtkanal 


\section{Diskussion und Ausblick}

Für die Entwicklung hochauflösender Scheinwerfer kommen verschiedene Technologien infrage. Alle Module mit subtraktiver Bilderzeugung wie ein LCD, DMD oder LCoS haben gemeinsam, dass der Nutzungsgrad einen direkt Einfluss auf die Systemeffizienz hat. Für die Umsetzung vollständiger KFZ-Lichtverteilungen mit einem typischen Aspektverhältnis und großen Gradienten der Beleuchtungsstärke ist der Nutzungsgrad sehr gering.

In diesem Beitrag werden Konzepte vorgestellt, mit denen es möglich ist vollständige Lichtverteilungen mit einem DMD-Scheinwerfer zu erzeugen. Eine vielversprechende Idee ist der Einsatz einer stark verzeichnenden Projektionsoptik. Es werden verschiedene Konzepte zur Umsetzung dieser Idee vorgestellt und der Ansatz einer radial verzeichnenden Optik als Prototyp eines hochauflösenden Scheinwerfers realisiert. Der Nutzungsgrad steigt im betrachteten Fall um einem Faktor von 5.

Die Simulation des Systems zeigt, dass mit dem gewählten Ansatz vollständige Lichtverteilungen dargestellt werden können. Der Kontrast des Systems wird mittels Raytracing untersucht, indem an drei Positionen der Lichtverteilung Pixel ausgeschaltet werden. Dabei zeigt sich, dass das Abschalten von 16 nebeneinanderliegenden Pixeln zu einem Kontrastverhältnis von knapp 15:1 führt. Dieser relativ geringe Kontrast geht auf die leichte Unschärfe der stark verzeichnenden Optik sowie die chromatische Aberration zurück, welche im prototypischen Aufbau nicht kompensiert wird. Die Kontraste, die mit dem realen Systemaufbau erzeugt werden können liegen unterhalb dieser Werte, was auf Fertigungs- und Justagetoleranzen sowie Streulicht im System zurückzuführen ist. Deshalb ist es nicht möglich, mit dem aktuellen Aufbau beispielsweise eine ECE-konforme Abblendlichtverteilung zu erzeugen.

Der vorgestellte Prototyp eines hochauflösenden Scheinwerfers auf Basis der DMD-Technologie in Kombination mit der verzeichnenden Projektionsoptik erzeugt vollständig adaptive Lichtverteilungen und wird an einem Versuchsträger zur Validierung im Verkehrsraum genutzt. Untersuchungsgegenstand ist zurzeit die Informationsprojektion auf die Fahrbahnoberfläche, welche sich mit dem System deutlich sichtbar umsetzen lässt. 


\section{Literaturangaben}

[1] Kloppenburg, G.; Wolf, A.; Lachmayer, R.: High-resolution vehicle headlamps: technologies and scanning prototype. Advanced Optical Technologies 5(2) (2016) 147155

[2] Pfullmann, N.; Thiel, A.; Thamm, M.; Plöger, R.; Kloppenburg, G.; Wolf, A.; Lachmayer, R.: From mechanical ADB systems to high resolution headlamps - new opportunities of novel headlight systems. Proc. Int. Symp. on Automotive Lighting 2017, 357-365

[3] Roth, R.; Wallaschek, J.; Kloppenburg, G.; Lachmayer, R.; Meyer, M.; Thomschke, S.: RGB-Laser Scanning Module for Onroad Projection. Proc. Int. Symp. on Automotive Lighting 2015, 385-394

[4] Hesse, H.: BMBF-Project VOLIFA 2020 - High resolution light distribution by using a LCD. Proc. Int. Symp. on Automotive Lighting 2015, 495-502

[5] Lee, T.; Park, G.: The Developement of the Automotive Headlamp using the DMD. Proc. Int. Symp. on Automotive Lighting 2011, 524-534

[6] Grötsch, S.; Pfeuffer, A.; Liebetrau, T.; Oppermann, H.; Brink, M.; Fiederling, R.; Möllers, I.; Moisel, J.: Integrated High Resolution LED Light Source in an AFS/ADB Headlamp. Proc. Int. Symp. on Automotive Lighting 2015, 241-250

[7] VDI-Richtlinie 2206: Entwicklungsmethodik für mechatronische Systeme. 06.2004

[8] Jürgens, C.: Der kontrastadaptive Scheinwerfer, TEWISS - Technik und Wissen GmbH Verlag, Garbsen, 2015

[9] ECE-Richtlinie 112: Einheitliche Bedingungen für die Genehmigung der Kraftfahrzeugscheinwerfer für asymmetrisches Abblendlicht und/oder Fernlicht, die mit Glühlampen und/oder LED-Modulen ausgerüstet sind. 22.08.2014

[10] Gut, C.: Laserbasierte hochauflösende Pixellichtsysteme., KIT Karlsruhe Diss. 2017

[11] Günther, A.: Entwicklung eines aktiven Kraftfahrzeugscheinwerferkonzepts mit DMD-Array., TU Berlin Diss. 2010

[12] Bhakta, V.; Ballard, B.: High resolution adaptive headlight using Texas Instruments DLP technology. Proc. Int. Symp. on Automotive Lighting 2015, 483-494

[13] Kauschke, R.: Systematik zur lichttechnischen Gestaltung von aktiven Scheinwerfern., Universität Paderborn Diss. 2006

[14] DE-P 102016103649A8

[15] DE-P 102016001103A1

[16] DE-P 102014013202B3

[17] US-P 20170205040A1

[18] DE-P 102014225246A1

[19] DE-P 102010048659A1

[20] US-P 20170192224A1

[21] DE-P 102016103717A1 